\title{
Comparison of public codes for Drell-Yan processes at NNLO accuracy ${ }^{\dagger}$
}

\author{
Zoltán Trócsányi, ${ }^{a, b, *}$ Sergey Alekhin, ${ }^{c, d}{ }^{2}$ dam Kardos $^{e}$ and Sven-Olaf Moch ${ }^{c}$ \\ ${ }^{a}$ Institute for Theoretical Physics, ELTE Eötvös Loránd University \\ Pázmány Péter 1/A, H-1117 Budapest, Hungary \\ ${ }^{b}$ ELKH-DE Particle Physics Research Group, University of Debrecen \\ 4010 Debrecen, PO Box 105, Hungary \\ ${ }^{c}$ Institute for High Energy Physics \\ 142281 Protvino, Moscow region, Russia \\ ${ }^{d}$ II. Institut für Theoretische Physik, Universität Hamburg \\ Luruper Chaussee 149, D-22761 Hamburg, Germany \\ ${ }^{e}$ Department of Experimental Physics, University of Debrecen \\ 4010 Debrecen, PO Box 105, Hungary \\ E-mail: trocsanyi.zoltan@ttk.elte.hu,
}

We present a detailed comparison of predictions at NNLO accuracy computed by four publicly available computer codes for Drell-Yan processes at the LHC and Tevatron colliders. We point out that while there is agreement among the predictions at the next-to-leading order accuracy, the predictions for fiducial cross sections at the next-to-next-to-leading order (NNLO) differ, whose extent depends on the observable. The sizes of the differences in general are at least similar, sometimes larger than the sizes of the NNLO corrections themselves.

\footnotetext{
*** The European Physical Society Conference on High Energy Physics (EPS-HEP2021), *** *** 26-30 July $2021 * * *$

*** Online conference, jointly organized by Universität Hamburg and the research center DESY ***
}

\footnotetext{
${ }^{\dagger}$ Research supported by the Hungarian National Research, Development and Innovation Office - NKFIH K 125105. ${ }^{*}$ Speaker
} 


\section{Introduction}

Drell-Yan lepton pair production is among the best-measured processes at the Large Hadron Collider (LHC). The experimental fiducial measurements of cross sections for the process proton + proton $\rightarrow V \rightarrow$ lepton pair where $V$ is a charged or neutral vector boson $\left(W^{ \pm}\right.$or $\left.Z^{0} / \gamma^{*}\right)$ have reached an accuracy below 1\% apart from the luminosity uncertainty (see ATLAS [1] and CMS [2] results obtained at $7 \mathrm{TeV}$ and $13 \mathrm{TeV}$ collider energies). Clearly, in order to utilize such data in particle phenomenology, in particular, in the determination of the parton distribution functions (pdf) at the next-to-next-to-leading order (NNLO) accuracy, the precision of the theoretical predictions is expected to match that of the experimental results.

The state of the art for predictions of fiducial cross sections in the Drell-Yan process in fixedorder QCD perturbation theory is formally at the NNLO accuracy [3-8]. These predictions have been tested and found to agree when computing the total cross section [9]. However, when the fiducial cross sections are compared [1,10] significant differences are observed in some regions of the phase space. The goal of this contribution is to exhibit those differences and offer some possible explanation for them.

To reach this goal, we focus on two aspects. For one, we provide benchmark numbers for NNLO QCD predictions in kinematics which are representative for the bulk of the available experimental data. We aim at providing predictions with a residual uncertainty below $\mathrm{O}(0.1 \%)$ in each bin of the distributions from the Monte Carlo integration for the cross section integrated over the fiducial region. Previous comparisons [9] of some of the published codes had limitations in the precision of the numerical integrations.

The second aspect concerns the investigation of the origin of the deviations among the codes from the point of view of the type of the subtraction schemes used for the regularization of the real emissions. These can be either local subtractions or global slicing, depending on whether the cancellations of the infrared singularities are performed locally in the integrand at each point in phase space or globally after integrating over a slice of the phase space. We illustrate the impact of fiducial cuts on the decay leptons for global slicing regularization methods.

\section{Comparison of public codes}

While the comparison of theoretical predictions does not strictly require experimental data sets, nevertheless, we find it interesting to show comparisons also to the experimental data, which may provide a better insight about the extent of the differences. Hence, in our comparisons we use the cuts that were employed in Ref. [1] in proton-proton collisions at $7 \mathrm{TeV}$ colliding energy. Those data include pseudo-rapidity distributions of the charged leptons (electron or muon in the decays of $W^{ \pm}$) and those of the lepton pairs emerging in the decay of neutral vector bosons. The transverse momenta $p_{T}^{\ell}$ and the pseudo-rapidities $\eta_{\ell}$ of the decay leptons were subject to fiducial cuts that we specify later. The cross sections for neutral boson production are measured at central as well as at forward pseudo-rapidities.

The other data were obtained by the D0 collaboration in proton-antiproton collisions at $1.96 \mathrm{TeV}$ colliding energy [11]. They measured the electron charge asymmetry and its dependence on the pseudo-rapidity of the electron. They published distributions obtained with both symmetric and 
staggered fiducial cuts on the transverse momenta and pseudo-rapidities of the electron and the neutrino, such that the data test forward kinematics, too.

Careful definition of the input parameters is important for a precision comparison. We used the $G_{\mu}$ scheme with input values $G_{\mathrm{F}}$ (Fermi constant), $M_{Z}, M_{W}$ and $\Gamma_{Z}, \Gamma_{W}$ (masses and widths of the vector bosons): $G_{\mathrm{F}}=1.16637 \times 10^{-5} \mathrm{GeV}^{-2}$,

$$
M_{Z}=91.1876 \mathrm{GeV}, \quad \Gamma_{Z}=2.4952 \mathrm{GeV}, \quad M_{W}=80.379 \mathrm{GeV}, \quad \Gamma_{W}=2.085 \mathrm{GeV} .
$$

In this scheme the weak mixing characterized by $\sin ^{2} \theta_{\mathrm{W}}$ and the electromagnetic coupling $\alpha\left(M_{Z}\right)$ are output values. It is useful in the sense that it minimizes the effect of the electroweak radiative corrections [12]. The relevant central values of the CKM matrix elements are

$$
\begin{array}{rlrl}
\left|V_{u d}\right|=0.97401, & \left|V_{u s}\right|=0.2265, & & \left|V_{u b}\right|=0.00361, \\
\left|V_{c d}\right|=0.2265, & \left|V_{c s}\right|=0.97320, & \left|V_{c b}\right|=0.04053 .
\end{array}
$$

We perform all computations in the $\overline{\mathrm{MS}}$ factorization scheme with $n_{f}=5$ light flavours, and with renormalization and factorization scales fixed at the mass of the relevant vector boson. In order to provide physical predictions we have to choose a particular pdf set. As this choice does not bias any of the codes in the comparison, we can choose it freely. We used the ABMP16 set $[13,14]$ with $\alpha_{s}^{\left(n_{f}=5\right)}\left(M_{Z}\right)=0.1147$.

We have selected the following publicly available codes for this study:

- DYNNLO (version 1.5) [3, 4] (available from http: //theory . fi .infn.it/grazzini/dy . html). This code uses $q_{T}$-subtraction, which is essentially a slicing method. The slicing parameter is called $r_{\text {cut }}$, representing a cut on $q_{T}$. The computation of pdf uncertainties is not included in this code.

- FEWZ (version 3.1) $[15,16]$ (available from https://www.hep.anl.gov/fpetriello/ FEWZ.html). This code employs a fully local subtraction method.

- MATRIX (version 1.0.4) [17] (available from https: //matrix . hepforge . org/). This code also uses $q_{T}$-subtraction. A drawback is that built-in computation of pdf uncertainties is not available. It uses the scattering amplitudes from OpenLoops [18].

- MCFM (version 9.0) [19] (available from https://mcfm. fnal.gov/). MCFM uses the $N$ jettiness subtraction [6], which is also a slicing method. The slicing parameter is a cut on jettiness, denoted by $\tau_{\text {cut }}$.

The consistent implementation of the parameters can be tested in a computation of the cross sections at leading-order in perturbation theory. We found agreement among all four predictions at a level of numerical precision $O\left(10^{-5}\right)$, which set the scene for comparisons including the effect of radiative corrections.

At the next-to-leading order (NLO) accuracy we found agreement among the predictions by FEWZ, MATRIX and MCFM. However, DYNNLO provides predictions that are accurate typically to a few per mille and deviate in particular for distributions with challenging kinematics and with deviations displaying a particular pattern as a function of the (di-)lepton pseudo-rapidities (see Ref. [10] for details). 

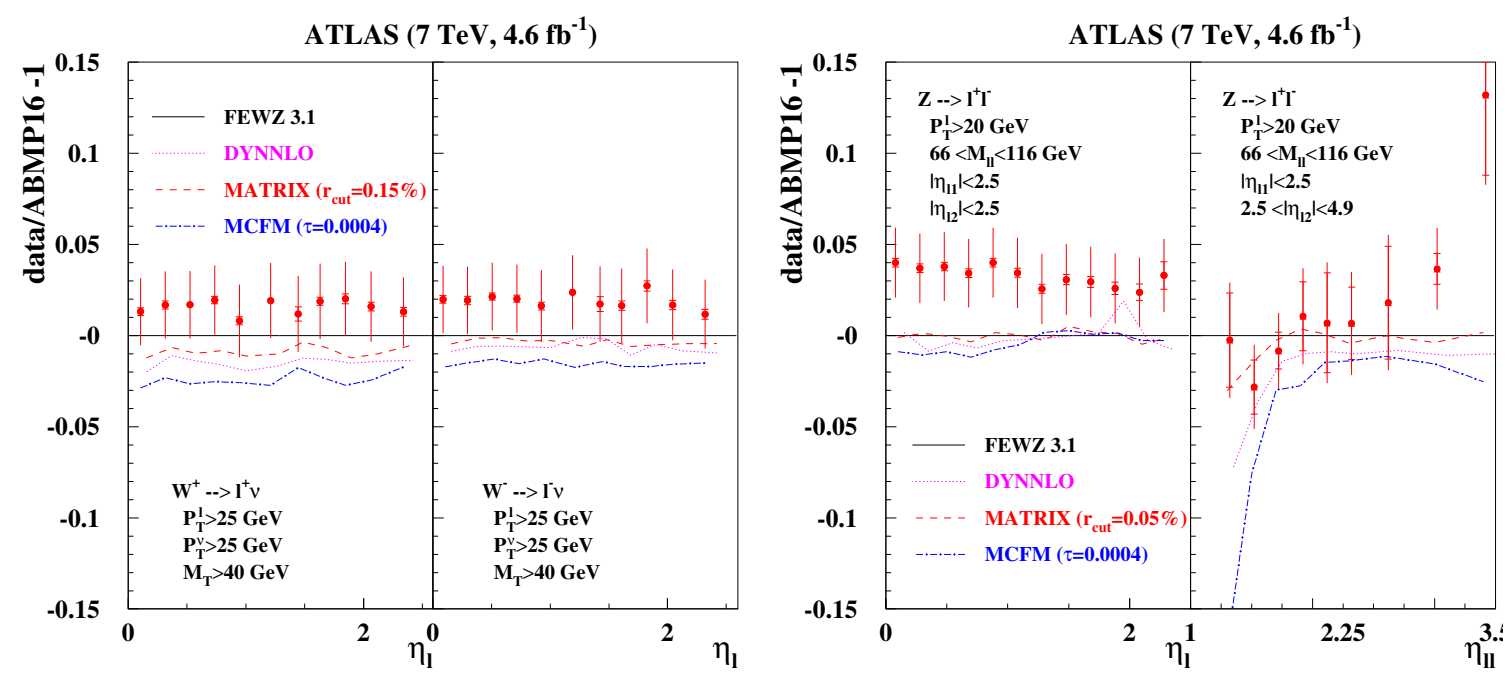

Figure 1: Compilation of the NNLO theory predictions (dotted: DYNNLO, dashed: Matrix, dash-dotted: MCFM) compared to ATLAS data shown with the statistical (inner bar) and the total uncertainties, including the systematic ones, at $\sqrt{s}=7 \mathrm{TeV}$ [1]. All plots used the ABMP16 pdf set and are normalized by the central predictions at NNLO obtained with FEWZ. The fiducial cuts on the decay leptons in the final state are indicated in the figure. Left plot: $p p \rightarrow W^{ \pm}+X \rightarrow l^{ \pm} v+X$ with slicing cuts $r_{\text {cut }}=0.15 \%$ in MATRIX and $\tau_{\text {cut }}=4 \cdot 10^{-4}$ in MCFM. Right plot: $p p \rightarrow Z / \gamma^{*}+X \rightarrow l^{+} l^{-}+X$ with $r_{\text {cut }}=0.05 \%$ for $Z \rightarrow l^{+} l^{-}$in MATRIX.

After validation we are ready to present the benchmark predictions of the public codes at the NNLO accuracy. Fig. 1 (left plot) shows the NNLO QCD cross sections for the inclusive lepton pair production in the process $p+p \rightarrow W^{ \pm}+X \rightarrow \ell^{ \pm} v+X$ as a function of the pseudo-rapidity of the charged lepton. The experimental cuts are as shown in the plots. The uncertainties in the cross sections from the numerical Monte Carlo integration have reached the target value of $10^{-4}$, hence negligible in all cases. The NNLO QCD corrections are small, $\mathrm{O}(1 \%)$ in most of the bins. Yet, the spread of the predictions by the four codes reaches $2-3 \%$, with the largest difference between FEWZ 3.1 and MCFM. Hence, the differences seen in the plots are at least comparable, often larger in size than the NNLO corrections themselves, which shows significant uncertainties in the computation of the NNLO corrections for these observables.

Interestingly, the predictions by the codes employing slicing fall below those of FEWZ 3.1. A similar observation can be made from Fig. 1 (right plot) where we present the NNLO cross sections for the inclusive charged lepton pair production as a function of the di-lepton pseudo-rapidity in the central region and also one lepton central while the other one forward. For central $Z / \gamma^{*}$-production the four predictions exhibit a smaller spread, typically of several per mill, and at most $1 \%$ between FEWZ 3.1 and MCFM below about $\eta_{l l} \leq 1.5$, while the predictions agree for larger values of $\eta_{l l}$.

For forward $Z / \gamma^{*}$-production the spread is up to $\mathrm{O}(1-2 \%)$ in the entire $\eta_{l l}$ range, but in the first bins where we see significant deviations, up to $\mathrm{O}(7 \%)$, which is clearly unacceptable. Similar systematic differences can also be observed in the electron charge asymmetry as a function of the pseudo-rapidity $\eta_{\mathrm{e}}$ of the electron, which in this case increases with $\eta_{\mathrm{e}}$. The details of these comparisons can be found in Ref. [10]. 


\section{Power corrections}

The observation of such differences among the predictions for Drell-Yan lepton pair production calls for an investigation of possible causes. The global slicing methods, such as $q_{T}$-subtraction or $N$-jettiness subtraction neglect power corrections. Hence, assuming that the implementations of these methods are correct in the public codes, the neglected power corrections offer a plausible explanation for the observed differences, which we study next.

The slicing parameter of the global slicing method - denoted generically here by $\tau$ - vanishes at Born level and resolves additional radiation in an infrared-safe manner. Thus the phase space integration for the cross section can be written as

$$
\sigma=\int \mathrm{d} \tau \frac{\mathrm{d} \sigma}{\mathrm{d} \tau}=\int^{\tau_{\text {cut }}} \mathrm{d} \tau \frac{\mathrm{d} \sigma}{\mathrm{d} \tau}+\int_{\tau_{\text {cut }}} \mathrm{d} \tau \frac{\mathrm{d} \sigma}{\mathrm{d} \tau}=\sigma\left(\tau_{\text {cut }}\right)+\int_{\tau_{\text {cut }}} \mathrm{d} \tau \frac{\mathrm{d} \sigma}{\mathrm{d} \tau},
$$

where $\tau_{\text {cut }}$ is the cut for the slicing of the phase space. According to the universal factorization of QCD in soft and collinear limits the dependence of $\mathrm{d} \sigma / \mathrm{d} \tau$ on $\tau$ has the structure

$$
\frac{\mathrm{d} \sigma}{\mathrm{d} \tau} \sim \delta(\tau)+\sum_{i}\left[\frac{\ln ^{i} \tau}{\tau}\right]_{+}+\sum_{j} \tau^{p-1} \ln ^{j} \tau+\mathrm{O}\left(\tau^{p}\right),
$$

where the +-distributions are the well-known leading threshold logarithms and the terms proportional to $\tau^{p-1}$ with $p>0$ are integrable and denote power corrections in the soft and collinear limit. Hence, after performing the integration, we may write schematically that

$$
\sigma\left(\tau_{\mathrm{cut}}\right) \sim 1+\sum_{i} \ln ^{i+1} \tau_{\mathrm{cut}}+\sum_{j} \tau_{\mathrm{cut}}^{p} \ln ^{j} \tau_{\mathrm{cut}}+\mathrm{O}\left(\tau_{\mathrm{cut}}^{p+1}\right)
$$

The power $p$ takes (i) positive integer values for the production of a stable gauge boson $V$, and (ii) half integers $p=1 / 2,1,3 / 2$ for subsequent decay with cuts on the leptonic final state.

The global subtraction schemes are implemented via a global subtraction term $\sigma^{\text {sub }}\left(\tau_{\text {cut }}\right)$ as

$$
\sigma=\sigma^{\mathrm{sub}}\left(\tau_{\text {cut }}\right)+\int_{\tau_{\text {cut }}} \mathrm{d} \tau \frac{\mathrm{d} \sigma}{\mathrm{d} \tau}+\Delta \sigma^{\mathrm{sub}}\left(\tau_{\text {cut }}\right)
$$

where the last term, $\Delta \sigma^{\text {sub }}\left(\tau_{\text {cut }}\right)=\sigma\left(\tau_{\text {cut }}\right)-\sigma^{\text {sub }}\left(\tau_{\text {cut }}\right)$, encodes the residual power corrections that are often neglected in slicing methods, resulting in an intrinsic error. Moreover, if the global subtraction term cancels only the leading soft and collinear singularities in $\sigma$, then the residual power corrections in the presence of cuts on the decay leptons are enhanced to linear power in $q_{T}$.

Neglecting the masses of the leptons, the lepton phase space - in terms of the variables $q_{T}$, the difference $\Delta y$ between the rapidity of $q$ and $p_{1}$, the azimuthal angle $\phi$ of $p_{1}-$ is

$$
\Phi_{L}\left(q_{T}\right)=\left(\int \prod_{i=1}^{2} \frac{\mathrm{d}^{4} p_{i}}{(2 \pi)^{3}} \delta^{+}\left(p_{i}^{2}\right)\right)(2 \pi)^{4} \delta^{(4)}\left(q-p_{1}-p_{2}\right)=\frac{1}{4 \pi^{2}} \int_{0}^{\pi} \mathrm{d} \phi \int_{-\infty}^{\infty} \mathrm{d} \Delta y \frac{p_{T 1}^{2}}{Q^{2}}
$$

where $q^{\mu}=p_{1}^{\mu}+p_{2}^{\mu}$, with $p_{1,2}^{\mu}$ being the lepton momenta and $Q^{2}=q^{2}$. After employing typical fiducial cuts, this phase space reads [10]

$$
\Phi_{L}\left(q_{T}\right)=\frac{1}{4 \pi^{2}} \int_{0}^{\pi} \mathrm{d} \phi \int_{-\infty}^{\infty} \mathrm{d} \Delta y \frac{p_{T 1}^{2}}{Q^{2}}\left(\prod_{i=1}^{2} \theta\left(p_{T i}-p_{T}^{\min }\right) \theta\left(\eta_{i}-\eta_{i}^{\min }\right) \theta\left(\eta_{i}^{\max }-\eta_{i}\right)\right) .
$$



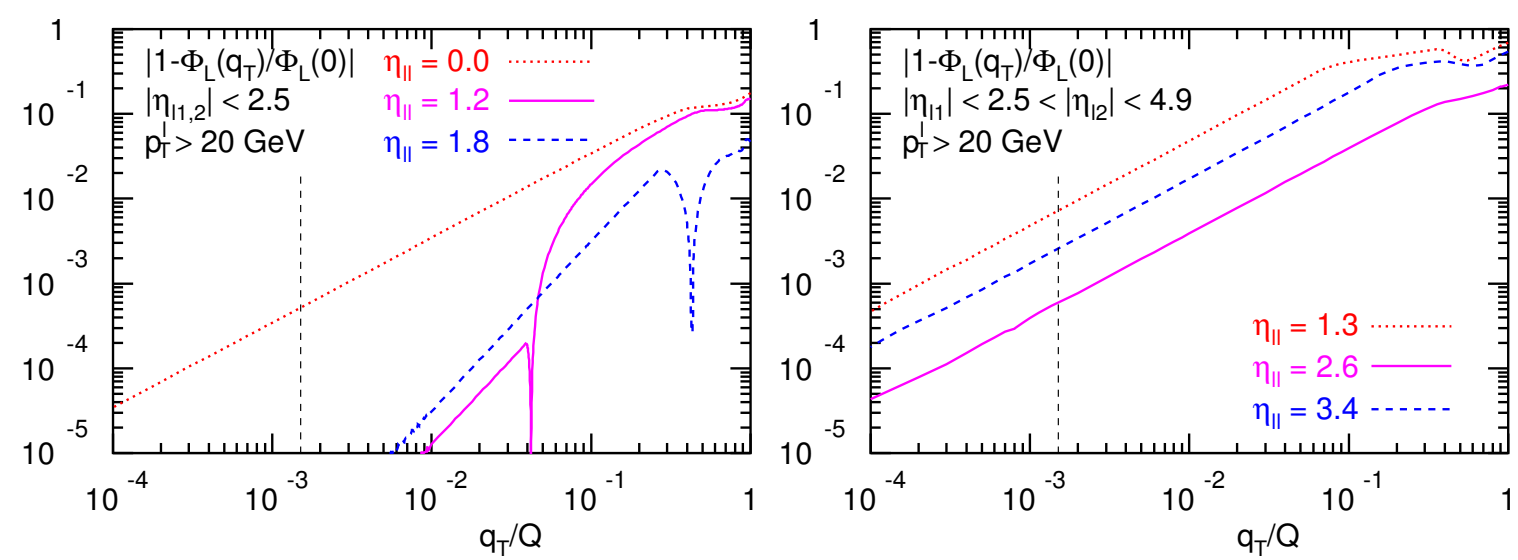

Figure 2: Difference between the Born and real emission phase spaces $\Phi_{L}(0)-\Phi_{L}\left(q_{T}\right)$ of the decay leptons relative to the Born one at fiducial cuts applied to ATLAS data set [1] for $Z / \gamma^{*}$-boson production $\left(Q=M_{Z}\right)$ for different values of the gauge boson pseudo-rapidity $\eta_{l l}$. For the lepton momenta $p_{T}^{l} \geq 20 \mathrm{GeV}$ are required. Left: Cuts selecting central pseudo-rapidities $\left|\eta_{l_{i}}\right| \leq 2.5$ for $i=1,2$. Right: Cuts selecting one lepton at central pseudo-rapidity $\left|\eta_{l_{1}}\right| \leq 2.5$ and the other at forward pseudo-rapidity, $2.5 \leq\left|\eta_{l_{2}}\right| \leq 4.9$. The vertical dashed line indicates the minimum value $r_{\text {cut }}^{\min }=0.15 \%$ used in MATRIX as a slicing cut.

The $\theta$-functions break azimuthal symmetry for $q_{T} \gtrsim q_{T}^{*}$ due to rapidity cuts where $q_{T}^{*}$ can be approximated as $q_{T}^{*} \approx Q \frac{|Y|}{\sinh \left(\eta^{\max }\right)}$ for small $Y$ [10], with $Y$ being the pseudo-rapidity of the vector boson. For $q_{T}<q_{T}^{*}$ the azimuthal symmetry is restored and the power corrections are quadratic. The transition between these two regions of $q_{T}$ is sharp up to corrections.

Such a sharp transition can clearly be seen in Fig. 2 (left) where the difference between the Born and real emission phase spaces $\Phi_{L}(0)-\Phi_{L}\left(q_{T}\right)$ of the decay leptons normalized to the Born one is shown for different values of the gauge boson pseudo-rapidity $\eta_{l l}$. The fiducial cuts are those applied to the ATLAS data set [1] for $Z / \gamma^{*}$-boson production $\left(Q=M_{Z}\right)$. The left plot corresponds to cuts selecting leptons with central pseudo-rapidities $\left|\eta_{l_{i}}\right| \leq 2.5$ for $i=1,2$ and we observe the presence of linear power corrections in $q_{T}$ for central gauge boson pseudo-rapidities $\eta_{l l} \lesssim 1$ due to constraints on $p_{T}$. For larger $\eta_{l l}$ the pseudo-rapidity constraint above dominates the phase space $\Phi_{L}$ and azimuthal symmetry is restored, resulting in quadratic power corrections in $q_{T}$ for small enough $q_{T}$. The right plot shows the case of one lepton at central pseudo-rapidity and the other at forward pseudo-rapidity. In this case, azimuthal symmetry is always broken by the constraint on $p_{T}$, and we observe linear power corrections. For the chosen values of $\eta_{l l}=1.3,2.6$ and 3.4 they amount to $O(7 \%), \mathrm{O}(0.6 \%)$ and $O(2 \%)$ at the value of $r_{\text {cut }}=0.15 \%$ in MATRIX, and the relatively large size of these corrections is remarkable. Moreover, their $\eta_{l l}$ dependence matches well with the pattern of the observed deviations of DYNNLO, MATRIX and MCFM from FEWZ in Fig. 1.

\section{Conclusions}

We have provided benchmark predictions at NNLO accuracy for Drell-Yan lepton pair production computed using four publicly available codes. We found significant (larger than the numerical uncertainty of the Monte Carlo integrations) differences among these predictions often comparable 
or larger than the NNLO corrections. We have pointed out that the fiducial cuts on the transverse momenta and pseudo-rapidities of the decay leptons lead to linear power corrections in the slicing parameter used in the codes employing global subtraction methods for the regularization of double real emission. The differences share certain patterns across the range of pseudo-rapidities in the considered distributions, which we found to correlate with the appearance of linear power corrections as a function of $q_{T}$. The continuous increase in the precision of the experimental measurements, the theory predictions are expected to provide cross sections at NNLO (or beyond) such that the systematic uncertainties due to choices of particular schemes or algorithms for the computation can be safely neglected in comparison to the experimental uncertainties.

\section{References}

[1] M. Aaboud et al. [ATLAS], Eur. Phys. J. C 77 (2017) 367 , arXiv:1612.03016.

[2] A. M. Sirunyan et al. [CMS], JHEP 12 (2019) 061, arXiv:1909.04133.

[3] S. Catani and M. Grazzini, Phys. Rev. Lett. 98 (2007) 222002, arXiv:hep-ph/0703012.

[4] S. Catani et al., Phys. Rev. Lett. 103 (2009) 082001, arXiv:0903.2120.

[5] R. Gavin, Y. Li, F. Petriello, and S. Quackenbush, Comput. Phys. Commun. 182 (2011) 2388 , arXiv:1011.3540.

[6] R. Boughezal et al., Eur. Phys. J. C77 (2017) 7, arXiv:1605.08011.

[7] S. Höche, Y. Li, and S. Prestel, Phys. Rev. D91 (2015) 074015, arXiv:1405.3607.

[8] S. Camarda et al., Eur. Phys. J. C 80 (2020) 251, arXiv:1910.07049, [Erratum: Eur.Phys.J.C 80 (2020) 440].

[9] S. Alioli et al., Eur. Phys. J. C77 (2017) 280, arXiv:1606.02330.

[10] S. Alekhin, A. Kardos, S. Moch and Z. Trócsányi, Eur. Phys. J. C81 (2021) 573, arXiv:2104.02400.

[11] D0, V. M. Abazov et al., Phys. Rev. D91 (2015) 032007, arXiv:1412.2862, [Erratum: Phys. Rev.D91 (2015) 079901].

[12] S. Dittmaier and M. Huber, JHEP 01 (2010) 060, arXiv:0911.2329.

[13] S. Alekhin, J. Blümlein, S. Moch, and R. Placakyte, Phys. Rev. D96 (2017) 014011, arXiv:1701.05838.

[14] S. Alekhin, J. Blümlein, and S. Moch, Eur. Phys. J. C78 (2018) 477 (2018), arXiv: 1803.07537.

[15] Y. Li and F. Petriello, Phys. Rev. D86 (2012) 094034, arXiv:1208.5967.

[16] R. Gavin, Y. Li, F. Petriello, and S. Quackenbush, Comput. Phys. Commun. 184 (2013) 208, arXiv:1201.5896.

[17] M. Grazzini, S. Kallweit, and M. Wiesemann, Eur. Phys. J. C78 (2018) 537, arXiv:1711.06631.

[18] F. Cascioli, P. Maierhofer, and S. Pozzorini, Phys. Rev. Lett. 108 (2012) 111601, arXiv:1111.5206.

[19] J. Campbell and T. Neumann, JHEP 12 (2019) 034, arXiv:1909.09117. 